\title{
Patient-Related Risk Factors for Postoperative Infection After Cholecystectomy
}

\author{
Gona Jaafar $^{1,2} \cdot$ Folke Hammarqvist $^{1} \cdot$ Lars Enochsson $^{3} \cdot$ Gabriel Sandblom $^{1}$
}

Published online: 20 June 2017

(C) The Author(s) 2017. This article is an open access publication

\begin{abstract}
Background The impact of patient-related risk factors on the incidence of postoperative infection after cholecystectomy is relatively unknown.

Aim The aim of this study was to explore potential patient-related risk factors for surgical site infection (SSI) and septicaemia following cholecystectomy.

Materials and methods All cholecystectomies registered in the Swedish national population-based register for Gallstone Surgery and Endoscopic Retrograde Cholangiopancreatography (GallRiks) 2006-2014 were identified. The study cohort was cross-matched with the Swedish National Patient Register in order to obtain data on patient history and postoperative infections. Simple and multiple logistic regression analyses were performed in order to assess the impact of various comorbidities on the risk for SSI and septicaemia.

Results A total of 94,557 procedures were registered. A SSI was seen following 5300 procedures (5.6\%), and septicaemia following 661 procedures $(0.7 \%)$. There was a significantly increased risk for SSI in patients with connective tissue disease (odds ratio [OR] 1.404, 95\% confidence interval [CI] 1.208-1.633), complicated diabetes (OR 1.435, CI 1.205-1.708), uncomplicated diabetes (OR 1.391, CI 1.264-1.530), chronic kidney disease (OR 1.788, CI 1.458-2.192), cirrhosis (OR 1.764, CI 1.268-2.454) and obesity (OR 1.630, CI 1.475-1.802). There was a significantly higher risk for septicaemia in patients with chronic kidney disease (OR 3.065, CI 2.120-4.430) or cirrhosis (OR 5.016, CI 3.019-8.336).

Conclusion and discussion Certain comorbidities have an impact on the risk for postoperative infection after cholecystectomy, especially SSI. This should be taken into account when planning the procedure and when deciding on prophylactic antibiotic treatment.
\end{abstract}

Gona Jaafar

gona.jaafar@karolinska.se

1 Department of Clinical Sciences, Intervention and Technology (CLINTEC), Centre for Digestive Diseases, Karolinska Institute, (Gastrocentrum) Karolinska University Hospital, 14186 Stockholm, Sweden

2 P03, Karolinska University Hospital-Solna, 17176 Stockholm, Sweden

3 Department of Surgical and Perioperative Sciences, Umeå University, Umeå, Sweden

\section{Background}

Cholecystectomy is one of the most common abdominal surgical procedures in the world. In most cases, it is performed without major risk for serious complication, although bile leakage and intraoperative contamination may lead to surgical site infection (SSI). Nevertheless, all risks must be taken into account when surgery is considered. The incidence of SSI after cholecystectomy was found to be $2.4 \%$ in a meta-analysis of studies on perioperative antibiotics in laparoscopic cholecystectomy [1]. In 
elective cholecystectomy, there is a low incidence of postoperative infection so the routine use of antibiotic prophylaxis (AP) is not recommended [2-4]. After cholecystectomy for acute cholecystitis, the risk is significantly higher [5]. The lack of internationally accepted guidelines concerning AP in cholecystectomy has led to divergent routines regarding the use of AP in surgery for acute cholecystitis. A better understanding of risk factors for postoperative infection may help in gaining consensus on antibiotic guidelines.

Risk factors related to the procedure itself are wellknown and have been thoroughly studied [6] [7]. The risk for postoperative infection related to comorbidity, on the other hand, is not as well understood. Postoperative infection prolongs time in hospital and has a negative effect on recovery and healthcare costs. AP has not been shown to decrease the risk for postoperative infection in elective cholecystectomy [5], and a register-based study on AP in acute cholecystectomy, found that it did not reduce the risk even when adjusting for confounders [1].

There may be other factors, not yet identified, that influence the risk for postoperative infection. Furthermore, we are not aware of any measure effective in preventing infection. A recent study showed, for instance, that abdominal drainage does not prevent intra-abdominal complications after laparoscopic cholecystectomy for acute cholecystitis [8].

\section{Aim}

The aim of this study was to investigate procedure- and patient-related risk factors associated with postoperative infection. The outcomes were surgical site infection (SSI) including intra-abdominal abscess, and septicaemia including cholangitis.

\section{Materials and method}

The study population was obtained from the GallRiks. GallRiks is a Swedish national quality register for gallstone surgery and ERCP, which began in May 2005 [9]. Since then, it has gradually reached a national coverage above $85 \%$. It is continuously validated, showing high accuracy and completeness of data [10]. By the end of 2014, more than 90,000 cholecystectomies had been registered in the GallRiks. The patient records of all patients registered in the GallRiks are reviewed 30 days postoperatively by a local coordinator in order to detect and register all postoperative complications. In any case where deviation from the expected course is noted, the coordinator either registers this as a complication or confers with the local surgeon responsible as to its nature.

Variables registered include indication for surgery (uncomplicated gallstone disease or complications secondary to gallstone disease such as cholecystitis, pancreatitis or obstructive jaundice due to common bile duct stone). Patients with uncomplicated gallstone disease are predominantly operated electively, whereas those with complications often undergo acute surgery.

The Swedish National Inpatient Register (IPR) is a part of the National Patient Register (NPR). It has had national coverage since 1987. All diagnoses are coded according to the International Classification of Diseases code (ICD code). In the NPR, 99\% of all discharges are registered, including ICD codes for all relevant diagnoses. In a validation study, the correctness of the entries was found to be 85-95\% [11].

All diagnoses in the postoperative course were retrieved from GallRiks as well as NPR based on ICD code registered by physician responsible for the discharge. SSI was defined as infection necessitating antibiotic treatment or percutaneous drainage in GallRiks, and/or ICD code T81.4 from NPR. Septicaemia was defined as cholangitis in Gallriks and/or cholangitis (ICD code K83.0) and sepsis (ICD code A40 and A41) in NPR. We preferred the term septicaemia since the criteria included positive blood culture, not necessarily including a systemic inflammatory response to the septicaemia [12].

All procedures performed from 2006-2014 were included in the study. Data on procedure-related risk factors and relevant patient-related risk factors were obtained from the GallRiks. Patient-related risk factors and comorbidities were extracted from Swedish IPR. Cross-checking between the GallRiks and the International Patient Register (IPR) was performed.

Simple and multiple logistic regression analyses were performed in order to analyse risk factors for SSI and septicaemia. The covariates retrieved from the GallRiks were age (younger or older than 70 years), gender, ASA (1 vs $>1$ ), indication for cholecystectomy (elective due to biliary colic as sole indication versus acute due to cholecystitis, pancreatitis or obstructive jaundice), method of approach (laparoscopic or open and converted), operation time (less or more than $120 \mathrm{~min}$ ), antibiotic treatment and accidental gallbladder perforation. The covariates extracted from the IPR were: history of connective tissue disease (ICD codes M05-M06, M31.5, M32-M34, M35.1, M35.3 and M36.0); diabetes mellitus (ICD codes E10-E14); chronic kidney disease(ICD codes N03.2-N03.7, N05.2N05.7, N18, N19, I12.0, I13.1, Z49.0-Z49.2, Z94.0, Z99.2); liver cirrhosis (ICD codes K70.3, K71.7, K74, I85); immunodeficiency (ICD codes D80-D89); and obesity 
Table 1 Simple logistic regression analyses for SSI and septicaemia with covariates indicated

\begin{tabular}{|c|c|c|c|c|c|c|c|c|c|c|}
\hline \multirow[t]{3}{*}{ Variable } & \multicolumn{5}{|c|}{ Surgical site infection } & \multicolumn{5}{|l|}{ Septicaemia } \\
\hline & \multicolumn{5}{|c|}{ Simple logistic regression analyses for SSI } & \multicolumn{5}{|c|}{ Simple logistic regression analyses for septicaemia } \\
\hline & $N$ & $\%$ & $P$ value & Odds ratio & CI & $N$ & $\%$ & $P$ value & Odds ratio & $\mathrm{CI}$ \\
\hline $\begin{array}{l}\text { Age, }>70 \text { years versus } \\
<70 \text { years }\end{array}$ & $1375 / 12,725$ & 10.8 & $<0.001$ & 2.405 & $2.254-2.565$ & $232 / 12,725$ & 1.8 & $<0.001$ & 3.524 & $3.000-4.139$ \\
\hline $\begin{array}{l}\text { Gender, male versus } \\
\text { female }\end{array}$ & $2255 / 31,068$ & 7.3 & $<0.001$ & 1.554 & $1.469-1.643$ & $341 / 31,068$ & 1.1 & $<0.001$ & 2.191 & $1.880-2.553$ \\
\hline ASA $>1$ versus 1 & $3493 / 45,385$ & 7.7 & $<0.001$ & 2.186 & $2.062-2.317$ & $501 / 45,385$ & 1.1 & $<0.001$ & 3.419 & $2.860-4.087$ \\
\hline $\begin{array}{l}\text { Indication for surgery, } \\
\text { gallstone pain or } \\
\text { complications of } \\
\text { gallstone disease }\end{array}$ & $3216 / 39,876$ & 8.1 & $<0.001$ & 2.214 & $2.092-2.343$ & $514 / 39,876$ & 1.3 & $<0.001$ & 4.844 & $4.031-5.822$ \\
\hline $\begin{array}{l}\text { Open approach, } \\
\text { including conversion } \\
\text { from laparoscopic to } \\
\text { open or laparoscopic }\end{array}$ & $1868 / 13,450$ & 13.9 & $<0.001$ & 3.705 & $3.490-3.934$ & $309 / 13,450$ & 2.3 & $<0.001$ & 5.705 & $4.881-6.669$ \\
\hline Op. time $>120 \mathrm{~min}$ & $1985 / 22,711$ & 8.7 & $<0.001$ & 1.991 & $1.879-2.110$ & $301 / 22,711$ & 1.3 & $<0.001$ & 2.756 & $2.360-3.219$ \\
\hline Antibiotic treatment & $2632 / 31,025$ & 8.5 & $<0.001$ & 2.130 & $2.015-2.253$ & $438 / 31,025$ & 1.4 & $<0.001$ & 4.319 & $3.661-5.095$ \\
\hline $\begin{array}{l}\text { Accidental gallbladder } \\
\text { perforation }\end{array}$ & $1868 / 27,490$ & 6.8 & $<0.001$ & 1.364 & $1.287-1.446$ & $201 / 27,490$ & 0.7 & 0.226 & 1.109 & $0.938-1.311$ \\
\hline $\begin{array}{l}\text { Connective tissue } \\
\text { disease }\end{array}$ & $216 / 2035$ & 10.6 & $<0.001$ & 2.044 & $1.770-2.360$ & $38 / 2035$ & 1.9 & $<0.001$ & 2.807 & $2.017-3.907$ \\
\hline Complicated diabetes & $166 / 1269$ & 13.1 & $<0.001$ & 2.587 & $2.192-3.052$ & $27 / 1269$ & 2.1 & $<0.001$ & 3.177 & $2.153-4.689$ \\
\hline Uncomplicated diabetes & $608 / 5283$ & 11.5 & $<0.001$ & 2.347 & $2.146-2.567$ & $97 / 5283$ & 1.8 & $<0.001$ & 2.943 & $2.368-3.657$ \\
\hline Chronic kidney disease & $123 / 788$ & 15.6 & $<0.001$ & 3.168 & $2.608-3.848$ & $33 / 788$ & 4.2 & $<0.001$ & 6.483 & $4.536-9.268$ \\
\hline Cirrhosis & $44 / 345$ & 12.8 & $<0.001$ & 2.476 & $1.802-3.402$ & $17 / 345$ & 4.9 & $<0.001$ & 7.531 & $4.597-12.338$ \\
\hline Immunodeficiency & $28 / 489$ & 5.7 & 0.904 & 1.024 & $0.698-1.501$ & $4 / 489$ & 0.8 & 0.752 & 1.173 & $0.437-3.146$ \\
\hline Obesity & $507 / 6173$ & 8.2 & $<0.001$ & 1.562 & $1.420-1.719$ & $46 / 6173$ & 0.7 & 0.653 & 1.072 & $0.793-1.447$ \\
\hline
\end{tabular}

(ICD code E86), according to the ICD codes retrieved from NPR.

The study was approved by the Regional Research Ethics Committee, Stockholm, Sweden, and data were processed without entering the patients' files.

\section{Result}

A total of 94,557 procedures were registered during the study period. The outcomes of the simple logistic analyses are shown in Table 1 . OR with a $95 \%$ confidence interval for SSI were significant for all covariates apart from immunodeficiency (Table 1). Regarding septicaemia, ORs were significant with $95 \%$ confidence interval for all covariates except accidental gallbladder perforation, immunodeficiency and obesity (Table 1).

Multivariate analyses with SSI and septicaemia as outcome variables were performed, with patient-related comorbidities as covariates, adjusted for age, gender, ASA, indication for surgery, method of approach, operation time, antibiotic treatment and accidental gallbladder perforation. Regarding SSI, the OR was significantly increased for connective tissue disease, complicated and uncomplicated diabetes, chronic kidney disease, cirrhosis and obesity but not for immunodeficiency (Table 2). The risk for septicaemia was significantly increased in cirrhosis and chronic kidney diseases but not with the other comorbidities (Table 2).

Septicaemia within 30 days postoperatively was registered in the IPR following 538 procedures $(0.6 \%)$. Postoperative septic cholangitis was registered following 175 procedures $(0.2 \%)$ in the GallRiks. For 63 procedures, sepsis and/or septic cholangitis were registered in the GallRiks as well as in the IPR. Surgical site infection or infection requiring antibiotics were registered in the GallRiks following 4835 of the procedures (5.2\%). Wound infections within 30 days postoperatively were registered after 1532 of the procedures (1.6\%). Surgical site infection or infection requiring antibiotics were registered in the GallRiks as well as in the IPR following 1136 of the procedures. 
Table 2 Multiple logistic regression analyses for patient-related risk factors after adjustment for other confounders

\begin{tabular}{|c|c|c|c|c|c|c|}
\hline \multirow[t]{2}{*}{ Conditions } & \multicolumn{3}{|c|}{ Multiple logistic analyses for SSI } & \multicolumn{3}{|c|}{ Multiple logistic analyses for septicaemia } \\
\hline & $P$ value & Odds ratio & CI & $P$ value & Odds ratio & $\mathrm{CI}$ \\
\hline Connective tissue disease & $<0.001$ & 1.404 & $1.208-1.633$ & 0.004 & 1.656 & $1.170-2.343$ \\
\hline Complicated diabetes & $<0.001$ & 1.435 & $1.205-1.708$ & 0.158 & 1.345 & $0.891-2.029$ \\
\hline Uncomplicated diabetes & $<0.001$ & 1.391 & $1.264-1.530$ & 0.023 & 1.313 & $1.039-1.660$ \\
\hline Chronic kidney disease & $<0.001$ & 1.788 & $1.458-2.192$ & $<0.001$ & 3.065 & $2.120-4.430$ \\
\hline Cirrhosis & 0.001 & 1.764 & $1.268-2.454$ & $<0.001$ & 5.016 & $3.019-8.336$ \\
\hline Immunodeficiency & 0.468 & 0.864 & $0.582-1.282$ & 0.916 & 1.055 & $0.391-2.847$ \\
\hline Obesitiy & $<0.001$ & 1.630 & $1.475-1.802$ & 0.261 & 1.196 & $0.875-1.635$ \\
\hline
\end{tabular}

\section{Discussion}

Although the comorbidity conditions studied in the present cohort had only a moderate impact on the risk for postoperative infection, the study indicates that certain patientrelated factors should be taken into account when planning surgery for gallstone disease. In the case of patient-related risk factors, the risk for infection may, to some extent, be reduced by AP. Although surgical technique is of crucial relevance, more so than any other preventive measure, risk factors related to the patient per se should not be neglected. We are aware of patient-related risk factors prior to surgery, and these must be taken into account when planning the procedure and optimising patient safety. By taking these factors into account, and using tailored AP and careful surgical technique, the risk for postoperative infection may be decreased.

In the present study, connective tissue disease, complicated and uncomplicated diabetes, chronic kidney disease, cirrhosis and obesity, when adjusted for gender, age, ASA $>1$, operation indication, open approach, prolonged operation time, antibiotic treatment and gallbladder perforation, were found to increase the risk for SSI. An increased risk for septicaemia was associated with chronic kidney disease and cirrhosis.

The rate of complications requiring surgical or percutaneous reintervetion following laparoscopic cholecystectomy is approximately $10 \%$. In a study on 4359 patients undergoing laparoscopic cholecystectomy, it was found that patients with cholecystitis have an increased risk for developing bile leakage, intra-abdominal abscess and pneumonia [7]. This was also confirmed in the present study where indications for acute operation such as complicated gallstone disease increased the risk for both SSI and septicaemia. Older patients ( $>70$ years) also have an increased risk for postoperative infection, which is in accordance with the results in the present study.

In elective laparoscopic cholecystectomy, there is generally low risk for postoperative infection. The routine use of AP was therefore not recommended in a Cochrane report from 2010 [13]. However, for patients with high risk factors for postoperative infection, AP should be considered even if a complicated procedure is not anticipated.

The decision to perform cholecystectomy may be a confounder for a preoperatively foreseen risk for surgical complications, including surgical site infection [9]. In the present study, open surgery or conversion to open surgery increased the risk for SSI and septicaemia by a factor of three and four, respectively.

Poorly controlled diabetes with postoperative hyperglycaemia has been reported to increase the risk for postoperative wound infection [14]. In the present study, both complicated and uncomplicated diabetes were associated with more than double the risk for SSI and septicaemia.

There are well-known risk factors for postoperative infection. The present study shows the risk for SSI to be increased by factors of three and five in patients with chronic kidney disease and cirrhosis, respectively. The indication for surgery should therefore be weighed against the risk of performing gallstone surgery on these patient groups. In cases where surgery is unavoidable, AP should be considered and meticulous care must be taken to avoid spillage of bile and haemorrhage.

Patient comorbidity, in particular liver cirrhosis, chronic kidney disease and obesity, should be taken into account when deciding on antibiotic prophylaxis. On the other hand, overuse of AP must also be avoided. With the increasing spread of antibiotic resistance, great efforts should be taken to avoid unnecessary use of AP. Culturedirected antibiotic treatment decreases the chance of inadequate AP in acute cholecystectomy, thereby contributing to the fight against antibiotic resistance.

The present study was based on the IPR as a source of data on previous medical history. Even if the register has a high completeness, not all relevant diagnoses may have been reported, e.g. immunodeficiency was defined from the ICD codes, without uniform criteria for immunological testing. Immunodeficiency would probably have been 
found to have a stronger impact if more data had been available. Furthermore, even if the GallRiks is continuously validated, there may be under-reporting of postoperative infections.

In conclusion, the present study shows that comorbidity, in particular liver cirrhosis, kidney disease and obesity, is important risk factor for SSI and septicaemia following cholecystectomy. Even if the risk factors investigated in the present study did not have as much impact as that seen with other risk factors, such as the presence of acute cholecystitis, conversion to open surgery, perioperative bleeding or bile leakage and patient-related factors should be taken into account when planning the procedure and when deciding on AP.

Acknowledgements The study was supported by a grant from the Olle Engkvist Research Foundation.

Open Access This article is distributed under the terms of the Creative Commons Attribution 4.0 International License (http://crea tivecommons.org/licenses/by/4.0/), which permits unrestricted use, distribution, and reproduction in any medium, provided you give appropriate credit to the original author(s) and the source, provide a link to the Creative Commons license, and indicate if changes were made.

\section{References}

1. Pasquali S, Boal M, Griffiths EA, Alderson D, Vohra RS (2016) Meta-analysis of perioperative antibiotics in patients undergoing laparoscopic cholecystectomy. Br J Surg 103(1):27-34 discussion 34

2. Chang WT, Lee KT, Chuang SC, Wang SN, Kuo KK, Chen JS et al (2006) The impact of prophylactic antibiotics on postoperative infection complication in elective laparoscopic cholecystectomy: a prospective randomized study. Am J Surg 191(6):721-725

3. Yildiz B, Abbasoglu O, Tirnaksiz B, Hamaloglu E, Ozdemir A, Sayek I (2009) Determinants of postoperative infection after laparoscopic cholecystectomy. Hepatogastroenterology 56(91-92):589-592

4. Darkahi B, Videhult P, Sandblom G, Liljeholm H, Ljungdahl M, Rasmussen IC (2012) Effectiveness of antibiotic prophylaxis in cholecystectomy: a prospective population-based study of 1171 cholecystectomies. Scand J Gastroenterol 47(10):1242-1246

5. Coccolini F, Catena F, Pisano M, Gheza F, Fagiuoli S, Di Saverio $S$ et al (2015) Open versus laparoscopic cholecystectomy in acute cholecystitis. Systematic review and meta-analysis. Int J Surg 18:196-204

6. Murphy MM, Ng SC, Simons JP, Csikesz NG, Shah SA, Tseng JF (2010) Predictors of major complications after laparoscopic cholecystectomy: surgeon, hospital, or patient? J Am Coll Surg 211(1):73-80

7. Donkervoort SC, Kortram K, Dijksman LM, Boermeester MA, van Ramshorst B, Boerma D (2016) Anticipation of complications after laparoscopic cholecystectomy: prediction of individual outcome. Surg Endosc 30:5388-5394

8. Park JS, Kim JH, Kim JK, Yoon DS (2015) The role of abdominal drainage to prevent of intra-abdominal complications after laparoscopic cholecystectomy for acute cholecystitis: prospective randomized trial. Surg Endosc 29(2):453-457

9. Enochsson L, Thulin A, Osterberg J, Sandblom G, Persson G (2013) The Swedish registry of gallstone surgery and endoscopic retrograde cholangiopancreatography (GallRiks): a nationwide registry for quality assurance of gallstone surgery. JAMA Surg 148(5):471-478

10. Rystedt J, Montgomery A, Persson G (2014) Completeness and correctness of cholecystectomy data in a national register-GallRiks. Scand J Surg SJS 103(4):237-244

11. Ludvigsson JF, Andersson E, Ekbom A, Feychting M, Kim JL, Reuterwall C et al (2011) External review and validation of the Swedish national inpatient register. BMC Public Health 11:450

12. Singer M, Deutschman CS, Seymour CW, Shankar-Hari M, Annane D, Bauer M et al (2016) The Third International consensus definitions for sepsis and septic shock (Sepsis-3). JAMA 315(8):801-810

13. Sanabria A, Dominguez LC, Valdivieso E, Gomez G (2010) Antibiotic prophylaxis for patients undergoing elective laparoscopic cholecystectomy. Cochrane Database Syst Rev 12:CD00 5265

14. Chuang SC, Lee KT, Chang WT, Wang SN, Kuo KK, Chen JS et al (2004) Risk factors for wound infection after cholecystectomy. J Formos Med Assoc Taiwan yi zhi 103(8):607-612 